Mechanismen massenspektrometrischer Fragmentierungsreaktionen, $X V^{1)}$

\title{
Sterische und konformative Effekte in den Massenspektren von 5-tert-Butyl-1,3-cyclohexandiolen und ihren Methyläthern
}

\author{
Hans-Fr. Grützmacher* und Reinhardt Asche \\ Institut für Organische Chemie und Biochemie der Universität Hamburg, \\ D-2000 Hamburg 13, Papendamm 6*)
}

Eingegangen am 27. November 1974

Die Massenspektren der drei Stereoisomeren 5 - 7 des 5-tert-Butyl-1,3-cyclohexandiols, der Monomethyläther 8-11 und der Dimethyläther 12-14 unterscheiden sich durch die für Derivate des 1,3-Cyclohexandiols charakteristischen, sterisch kontrollierten Eliminierungsreaktionen von $\mathrm{H}_{2} \mathrm{O}, \mathrm{CH}_{2} \mathrm{O}$ und $\mathrm{CH}_{3} \mathrm{OH}$. Für 7 und seine Derivate 11 und 14 wird außerdem spezifisch die Abspaltung des tert-Butylsubstituenten als $\mathrm{C}_{4} \mathrm{H}_{8}$ beobachtet. - Die Analyse der massenspektrometrischen Fragmentierungen mit Hilfe deuterierter Derivate zeigt, daß die Molekül-Ionen von 5 und 6 sowie deren Derivate ausschließlich in der Grundkonformation fragmentieren, während die Molekül-Ionen von 7 und seinen Derivaten vor der Fragmentierung Konformationsänderungen erleiden. Dieses unterschiedliche Verhalten wird durch eine Konkurrenz zwischen schneller sterisch kontrollierter Fragmentierung und Konformationsänderung der Molekuil-Ionen erklärt.

\section{Mechanism of Mass Spectrometric Fragmentations, $X^{11}$}

Steric and Conformational Effects in the Mass Spectra of 5-tert-Butyl-1,3-cyclohexanediol and its Methyl Ethers

The mass spectra of the three stereoisomers 5-7 of 5-tert-butyl-1,3-cyclohexanediol, of the monomethyl ethers 8-11, and of the dimethyl ethers $12-14$ can be distinguished by steric controlled eliminations of $\mathrm{H}_{2} \mathrm{O}, \mathrm{CH}_{2} \mathrm{O}$, and $\mathrm{CH}_{3} \mathrm{OH}$ which are typically for 1,3-cyclohexanediols. Furthermore an elimination of the tert-butyl substituent as $\mathrm{C}_{4} \mathrm{H}_{8}$ is observed specifically in the mass spectra of 7 and its derivatives 11 and 14. - An analysis of the mass spectrometric fragmentation of deuterated derivatives shows, that the molecular ions of 5 and 6 and of their derivatives fragment exclusively from the ground conformation, while the molecular ions of 7 and its derivatives change their conformations before fragmentation. This difference in the behaviour of the molecular ions is explained on the basis of concurrent stereospecific fragmentation and changes in the conformation.

Die massenspektrometrische Untersuchung von cyclischen und bicyclischen Diolen ${ }^{2)}$ hat gezeigt, daß zwischen dem Fragmentierungsmuster der Molekül-Ionen und der räumlichen Anordnung der Hydroxygruppen bei den Stereoisomeren dieser Verbindun-

*) Neue Anschrift: Fakultät für Chemie, Universität Bielefeld, Postfach 8640 .

1) XIV. Mitteil.: D. Stefanovič und H.F. Grützmacher, Org. Mass Spectrom., im Druck.

2) 2a) H.F. Grützmacher und J. Winkler, Org. Mass Spectrom. 3, $1139(1970) .-{ }^{2 b)}$ H. F. Grützmacher und $K$. H. Fechner, Tetrahedron 27, 5011 (1971). 
gen eine eindeutige Beziehung besteht, die auf reaktive intramolekulare Wechselwirkungen zwischen den Carbinol-Gruppierungen der Molekül-Ionen zurückgefïhrt werden kann. Derartige sterische Effekte sind besonders leicht in den Massenspektren der Methyläther von cyclischen 1,3- und 1,4-Diolen zu beobachten, so daß sich diese Derivate gut für eine massenspektrometrische Identifizierung der Stereoisomeren eignen. Bei trans-Anordnung zweier Methoxygruppen in 1,3- oder 1,4-Stellung enthält das Massenspektrum einen großen Peak für $\left[\mathrm{M}-\mathrm{CH}_{3} \mathrm{OH}\right]^{\ddagger}$, während bei cis-Anordnung der Methoxygruppen neben einem merklich kleineren Peak für $\left[\mathrm{M}-\mathrm{CH}_{3} \mathrm{OH}\right]^{+}$zwei zusätzliche charakteristische Peaks fuir die Ionen $\left[\mathrm{M}-\mathrm{CH}_{2} \mathrm{O}\right]^{ \pm}$und $\left[\mathrm{M}-\mathrm{CH}_{2} \mathrm{O}-\mathrm{CH}_{3} \mathrm{OH}\right]^{ \pm}$im Spektrum beobachtet werden.

Durch die Analyse der Massenspektren spezifisch deuterierter Derivate konnte nachgewiesen werden, daß die Unterschiede durch Zusammenwirken sterischer und energetischer Effekte bei dem Zerfall der stereoisomeren Molekül-Ionen entstehen (Schema 1). So stehen sich z. B. in den Molekül-Ionen des trans-1,3-Dimethoxycyclohexans (1) eine Methoxygruppe und das H-Atom der anderen Carbinolgruppe auf relativ kurzem Abstand in einer 1,3-diaxialen Anordnung gegenüber und werden leicht als Methanol abgespalten, weil die Dissoziationsenergie der $\mathbf{C}-\mathbf{H}$-Bindung in der Carbinolgruppe ca. $20 \mathrm{kcal} / \mathrm{mol}$ kleiner ist als die der übrigen $\mathrm{C}-\mathrm{H}-$ Bindungen $^{3)}$. Bei den Molekül-Ionen des cis-1,3-Dimethoxycyclohexans (2) ist diese energetisch günstige Fragmentierung sterisch nicht möglich, so daß Methanol aus den Molekül-Ionen nur mit größerem Energieaufwand in einer unspezifischen Reaktion eliminiert werden kann. Dafür ist in einer der Konformationen der Molekül-Ionen von 2 bei 1,3-diaxialer Stellung der beiden Methoxygruppen ein kurzer Abstand zwischen den Substituenten vorhanden, der die im Schema 1 gezeigte intramolekulare H-Übertragung und die anschließende Reaktionssequenz zu den Ionen $\left[\mathrm{M}-\mathrm{CH}_{2} \mathrm{O}\right]^{\dagger}$ und $[\mathrm{M}-$ $\left.\mathrm{CH}_{2} \mathrm{O}-\mathrm{CH}_{3} \mathrm{OH}\right]^{ \pm}$ermöglicht.

Schema 1
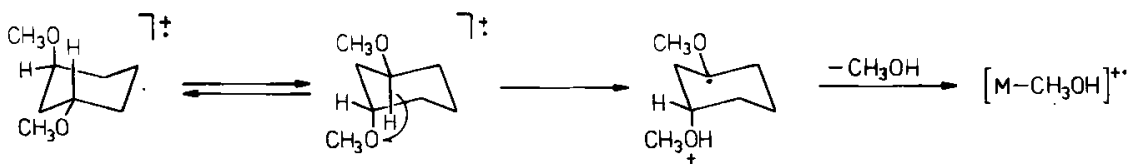

1
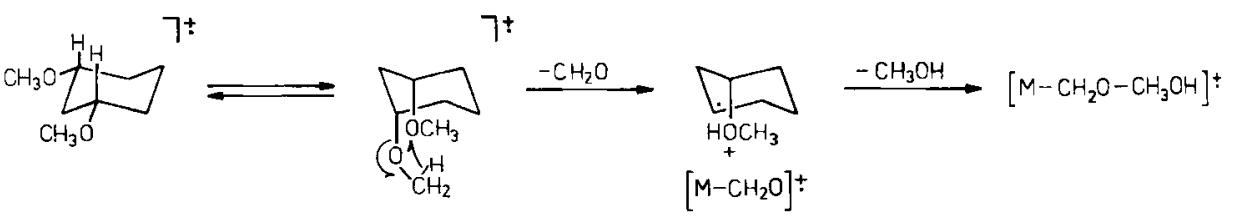

2

Die Konformation mit diaxialer Stellung der Methoxygruppen von 2 ist gegenüber der Konformation mit äquatorialer Anordnung der Substituenten energetisch benachteiligt. Die angeführte Erklärung für die Unterschiede in den Massenspektren von 1 und 2 setzt daher voraus, daß die Molekül-Ionen konformativ beweglich sind und auch energetisch ungünstige Konformationen erreicht werden können. Zu der gleichen Annahme zwingt auch die unterschiedliche Fragmen-

${ }^{3)}$ R. H. Martin, F. W. Lampe und R. W. Taft, J. Amer. Chem. Soc. 88, 1353 (1966).

Chemische Berichte Jahrg. 108 
tierung von Molekül-Ionen anderer stereoisomerer Cycloalkan- und Decalindiol-Derivate ${ }^{2)}$. Da nach der Ionisation durch Elektronenstoß die Überschußenergie in den Molekül-Ionen einige eV betragen kann und die Aufenthaltsdauer der Molekül-Ionen in der Ionenquelle des Massenspektrometers mit ca. $10^{-6} \mathrm{~s}$ groß genug ist für Konformationsänderungen von Cyclohexanderivaten, ist die Annahme einer konformativen Beweglichkeit der Molekül-Ionen plausibel. Gleichwohl liegen Beobachtungen vor, die diese Annahme in Frage stellen ${ }^{4)}$.

Zur Prüfung, welchen Einfluß die Molekülkonformationen auf die Massenspektren von Cyclohexandiolen haben können, wurden die Stereoisomeren von 5-tert-Butyl-1,3-cyclohexandiol, die entsprechenden Monomethyläther und die Dimethyläther hergestellt und massenspektrometrisch untersucht. 5-tert-Butyl-1,3-cyclohexandiol bietet sich für diese Untersuchungen an, weil durch den tert-Butylsubstituenten zumindest für die neutralen Moleküle auch unter den massenspektrometrischen Versuchsbedingungen die Konformation weitgehend fixiert ist.

\section{Darstellung der 5-tert-Butyl-1,3-cyclohexandiole und ihrer Methyläther}

Für die Synthese des 5-tert-Butyl-1,3-cyclohexandiols wurde ein Weg gesucht, der zu allen drei Stereoisomeren 5-7 führt und die Synthese spezifisch mit Deuterium markierter Derivate sowie der Monomethyläther 8-11 und der Dimethyläther 12-14 erlaubt. Dieses Ziel läBt sich durch den fuir 5 und 6 in der Literatur ${ }^{5)}$ angegebenen Syntheseweg gut erreichen. Das dritte Isomere 7 kann aus 6 durch Isomerisierung mit Raney-Nickel ${ }^{6}$ ) erhalten werden. Ausgangssubstanzen für die Synthesen sind die trans- und cis-Isomeren 3 und 4 des 5-tert-Butyl-2-cyclohexenols, die durch Bromierung von 4-tert-Butylcyclohexen mit $N$-Bromsuccinimid und anschließende Hydrolyse erhalten werden können. Das trans-Produkt 3 kann durch fraktionierte Kristallisation der $p$-Nitrobenzoesäureester aus dem Gemisch isoliert werden, während das cis-Isomere 4 durch Oxidation des Isomerengemisches der tert-Butylcyclohexenole zum Keton und Reduktion mit $\mathrm{LiAlH}_{4}$ zugänglich ist.

Schema 2

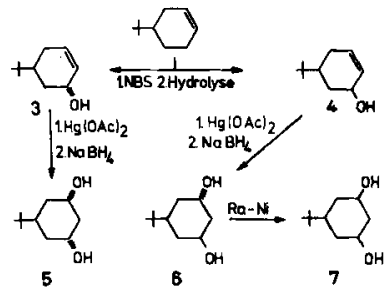

Die zweite Hydroxygruppe wird durch reduktive Oxymercurierung ${ }^{7)}$ in 3 und 4 bevorzugt in axialer Stellung eingefuhrt. Aus 3 entsteht daher bevorzugt das 5t-tert-Butyl$1 r, 3 c$-cyclohexandiol (5), aus 4 das 5c-tert-Butyl-1r,3t-cyclohexandiol (6). Die Reinigung der Rohprodukte gelingt nach Überführung in die Diacetate durch Säulenchromatogra-

\footnotetext{
4) $J$. Winkler, Dissertation, Univ. Hamburg 1970.

5) R. J. Ferrier und N. Prasad, J. Chem. Soc. B 1967,1417.

6) G. Rüdiger, Dissertation, Univ. Hamburg 1969.

7) P. Chamberlain und G. H. Witham, J. Chem. Soc. B 1970, 1382.
} 
phie. $5 c$-tert-Butyl-1r,3c-cyclohexandiol (7) kann aus 6 durch Isomerisierung mit Raney-Ni und säulenchromatographische Trennung des Isomerengemisches aus 6 und 7 gewonnen werden.

Die Monomethyläther 8 und 9 lassen sich aus 3 durch $O$-Methylierung und anschlieBende reduktive Oxymercurierung erhalten. Das Isomerengemisch enthält 8 und 9 im Verhältnis von ca. $4: 1$. Die Auftrennung wird durch Säulenchromatographie an Kieselgel erreicht. Analog wurde 3t-tert-Butyl-5t-methoxy-1r-cyclohexanol (10) aus 4 erhalten. Das in der Reihe der Monomethyläther noch ausstehende Isomere 11 konnte nicht als Nebenprodukt der Oxymercurierung erhalten werden, sondern mußte aus 10 durch Oxidation zum Keton, Reduktion mit $\mathrm{LiAlH}_{4}$ zu einem Gemisch aus 10 und 11 (ca. 1 :9) und Säulentrennung hergestelit werden.

Schema 3

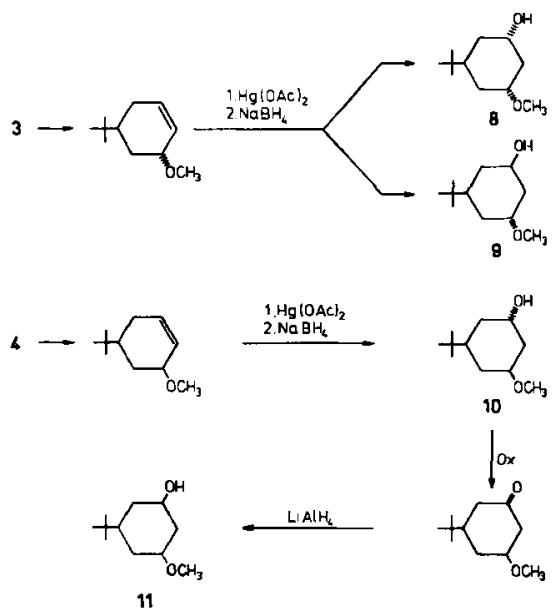

\section{Schema 4}

$$
\begin{aligned}
& \underbrace{O R^{1}}_{R^{4}} T^{O R^{2}} \\
& R^{1} 0 \underbrace{R^{3}}_{R^{4}} \underbrace{2} \\
& \left.R^{1} 0\right|_{R^{2}} ^{R^{3}}+ \\
& \begin{array}{c|cccc} 
& R^{1} & \mathbf{R}^{2} & \mathbf{R}^{3} & \mathbf{R}^{4} \\
\hline 5 & H & H & H & H \\
5-0-d_{2} & \mathrm{D} & \mathrm{D} & \mathrm{H} & \mathrm{H} \\
8 & \mathrm{CH}_{3} \mathrm{H} & \mathrm{H} & \mathrm{H} \\
8-0-\mathrm{d}_{1} & \mathrm{CH}_{3} & \mathrm{O} & \mathrm{H} & \mathrm{H} \\
8-\mathrm{d}_{1} & \mathrm{CH}_{3} \mathrm{H} & \mathrm{H} & \mathrm{D} \\
12 & \mathrm{CH}_{3} \mathrm{CH}_{3} & \mathrm{H} & \mathrm{H} \\
12-d_{1} & \mathrm{CH}_{3} \mathrm{CH}_{3} \mathrm{H} & \mathrm{D}
\end{array}
\end{aligned}
$$

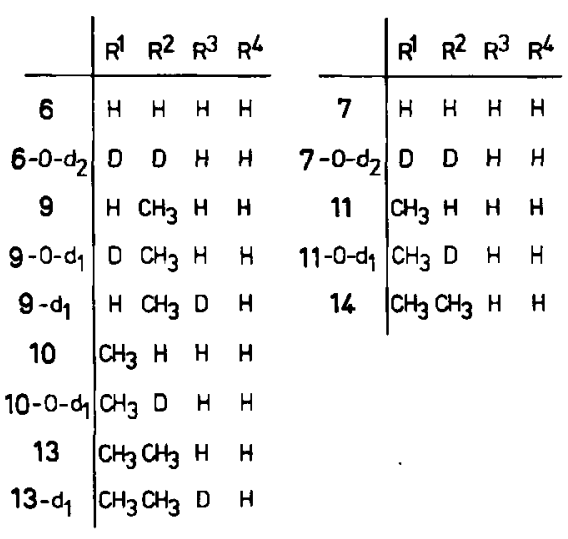


Die Dimethyläther 12-14 wurden aus den entsprechenden Monomethyläthern durch Methylierung mit Diazomethan/BF ${ }_{3}$ - ̈̈therat und Reinigung durch Säulenchromatographie erhalten. Zur Synthese der spezifisch an C-1 deuterierten Mono- und Dimethyläther wurde 8 zum Keton oxidiert und mit $\mathrm{LiAlD}_{4}$ zu einem Gemisch der deuterierten Isomeren 8- $d_{1}$ und 9- $d_{1}$ reduziert. Säulenchromatographische Trennung lieferte die reinen deuterierten Monomethyläther, aus denen durch Methylierung die entsprechenden Dimethyläther erhalten wurden.
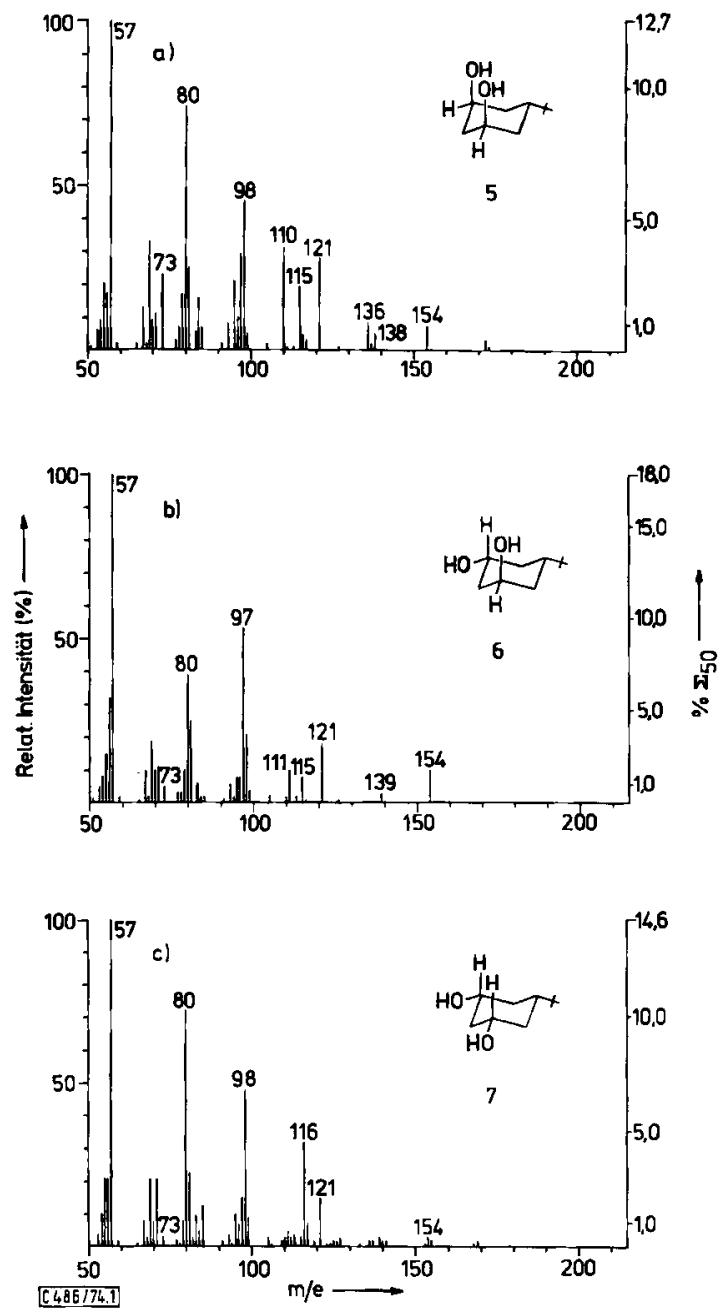

Abb. 1. Massenspektren der 5-tert-Butyl-1,3-cyclohexandiole, $70 \mathrm{eV},{ }^{13} \mathrm{C}$-korrigiert
a) 5 -tert-Butyl-1 $r, 3 c$-cyclohexandiol (5)
b) $5 c$-tert-Butyl-1r,3t-cyclohexandiol (6)
c) $5 c$-tert-Butyl-1r,3c-cyclohexandiol(7) 


\section{Massenspektren}

a) Diole

In Abb. 1 sind die Massenspektren der freien Diole 5-7 wiedergegeben. Wie für geometrische Isomere zu erwarten ist, unterscheiden sich die drei Massenspektren. Man beobachtet jedoch bei allen drei Verbindungen einen weitgehenden Abbau der Molekül-Ionen zu kleinen Fragmenten, für die es schwierig ist, den Ursprung im Molekül und den Zusammenhang ihrer Bildungsreaktionen mit der Molekülgeometrie eindeutig zu bestimmen. Nach den Erfahrungen, die über das massenspektrometrische Verhalten von 1,3-Cyclohexandiolen ${ }^{8)}$ und von 4-tert-Butyl- 9) bzw. 3-tert-Butylcyclohexanolen ${ }^{10)}$ vorliegen, läßt sich der Einfluß der Molekülgeometrie und der Konformation der Molekül-Ionen besonders deutlich an den $\left[\mathrm{M}-\mathrm{H}_{2} \mathrm{O}\right]^{\dagger}$-Ionen und deren Folgeprodukten erkennen.

Die $\left[\mathrm{M}-\mathrm{H}_{2} \mathrm{O}\right]^{\dagger}$-Ionen liefern in den Massenspektren von 5-7 den Peak bei $\mathrm{m} / \mathrm{e}=$ 154. Der Intensitätsabfall dieses Peaks in der Reihe $6 \rightarrow 5 \rightarrow 7$ entspricht der Erwartung für eine weitgehende sterische Kontrolle der Wassereliminierung aus den MolekülIonen, denn bei 6 ist durch die 1,3-diaxiale Anordnung einer OH-Gruppe und eines Carbinol-H-Atoms die Wasserabspaltung sterisch und energetisch begünstigt, bei 5 ist die Eliminierung von $\mathrm{H}_{2} \mathrm{O}$ durch eine intramolekulare Reaktion zwischen beiden HO-Gruppen sterisch ohne Änderung der Molekülkonformation möglich, während bei 7 eine Wassereliminierung nur nach einer Konformationsänderung oder Aufspaltung des cyclischen Molekül-Ions eintreten kann. Diese Erklärung wird durch die Massenspektren der 5-tert-Butyl-1,3-cyclohexandiole-O- $\mathrm{d}_{2}$ bestätigt (Tab. 1). Die Molekül-Ionen von 6-O-

Tab. 1. Intensitätsverteilung der Abspaltungen von $\mathrm{H}_{2} \mathrm{O} / \mathrm{HDO} / \mathrm{D}_{2} \mathrm{O}$ und $\mathrm{CH}_{3} \mathrm{OH} / \mathrm{CH}_{3} \mathrm{OD}$ in den Massenspektren deuterierter Derivate von Cyclohexandiolen und ihren Methyläthern

\begin{tabular}{|c|c|c|c|c|c|}
\hline$[\mathrm{M}$ & $\underset{(\%)}{\left.-\mathrm{H}_{2} \mathrm{O}\right]^{+}}$ & {$\left[\begin{array}{c}\mathrm{M}-\mathrm{HDO}]^{ \pm} \\
(\%)\end{array}\right.$} & $\underset{(\%)}{\left[M-\mathrm{D}_{2} \mathrm{O}\right]^{+}}$ & {$\left[\mathrm{M}-\underset{(\%)}{\left.\mathrm{CH}_{3} \mathrm{OH}\right]}\right.$} & {$\left[\mathrm{M}-\underset{(\%)}{\mathrm{CH}_{3} \mathrm{OD}}\right]^{+}$} \\
\hline $5-\mathrm{O}-\mathrm{d}_{2}$ & 0 & 30 & 70 & - & - \\
\hline $6-\mathrm{O}-\mathrm{d}_{2}$ & 0 & 95 & 5 & - & - \\
\hline $7-O-d_{2}$ & b) & b) & b) & - & - \\
\hline $8-O-d_{1}$ & 0 & 100 & - & 33 & 67 \\
\hline $8-d_{1}$ & 100 & 0 & - & 100 & 0 \\
\hline $9-0-d_{1}$ & 0 & 100 & - & 100 & 0 \\
\hline $9-d_{1}$ & 100 & 0 & - & $<5$ & $>95$ \\
\hline $10-O-d_{1}$ & 0 & 100 & - & 100 & 0 \\
\hline $11-0-d_{1}$ & 0 & 100 & - & b) & b) \\
\hline $12-\mathrm{d}_{1}$ & - & - & - & 70 & 30 \\
\hline 13-d & - & - & - & 10 & 90 \\
\hline
\end{tabular}

a) Auf $100 \%$ für die Intensität der Peak-Gruppe normiert.

b) Intensität der Peak-Gruppe zu gering.

$d_{2}$ verlieren ausschlieBlich $\mathrm{HDO}$, während für 5-O- $\mathrm{d}_{2}$ zu $70 \%$ die Eliminierung von $\mathrm{D}_{2} \mathrm{O}$ durch Reaktion zwischen den beiden OD-Gruppen und nur zu 30\% eine Abspaltung von HDO beobachtet wird, in Ubereinstimmung mit dem Massenspektrum von cis-1,3-

8) H. F. Grützmacher, Suom. Kemistilehti A 46, 50 (1973) [C. A. 79, 4530 b (1973)].

9) Z. M. Akhtar, C. E. Brion und L. D. Hall, Org. Mass Spectrom. 8, 189 (1974).

10) L. Dolejs und V. Hanus, Collect. Czech. Chem. Commun. 33, 332 (1968). 
Cyclohexandiol-O- $\mathrm{d}_{2}{ }^{8}$. Die besondere Bildungsreaktion und Struktur der Mehrzahl $\operatorname{der}\left[\mathrm{M}-\mathrm{H}_{2} \mathrm{O}\right]^{ \pm}$-Ionen bei 5 erklärt auch die Bildung der für 5 typischen Ionen bei $m / e=$ 110 durch eine nachfolgende McLafferty-Umlagerung:

Schema 5

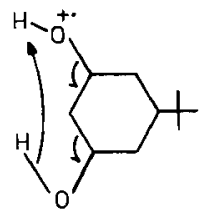

$5^{7+}$

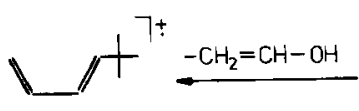

me 110
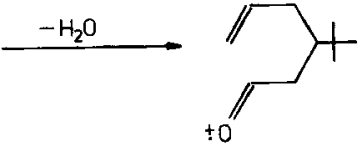

mle 154

|ll

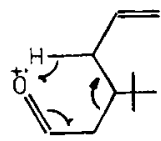

Im Massenspektrum von 7-O- $\mathrm{d}_{2}$ beobachtet man ebenfalls bevorzugt die Eliminierung von $\mathrm{D}_{2} \mathrm{O}$. Die Intensität der Ionen $\left[\mathrm{M}-\mathrm{H}_{2} \mathrm{O}\right]^{\ddagger}$ bzw. $\left[\mathrm{M}-\mathrm{D}_{2} \mathrm{O}\right]^{\ddagger}$ ist jedoch gering. $\mathrm{Da}$ außerdem die Möglichkeit berücksichtigt werden muß, daß aus den Molekül-Ionen von 7-O-d $\mathrm{d}_{2}$ nach einer Aufspaltung des Cyclohexanringes $\mathrm{D}_{2} \mathrm{O}$ eliminiert wird, kann dieses Ergebnis nur als Hinweis, nicht als Beweis dafür gelten, daß die Molekül-Ionen von 7 vor der Fragmentierung von der Grundkonformation in eine energiereichere Konformation übergehen können.

Es gibt jedoch einen weiteren deutlichen Hinweis auf Konformationsänderungen der Molekül-Ionen von 7 vor dem Zerfall. Das Massenspektrum von 7 zeichnet sich durch einen Peak der $\left[\mathrm{M}-\mathrm{C}_{4} \mathrm{H}_{8}\right]^{ \pm}$-Ionen bei $m / e=116$ aus, während bei 5 und 6 bejorzugt die Abspaltung von $\mathrm{C}_{4} \mathrm{H}_{9}$ zu Ionen $m / e=115$ eintritt. In der Reihe der tert-Butylcyclohexanol-Derivate ist die Eliminierung von $\mathrm{C}_{4} \mathrm{H}_{8}$ aus den Molekül-Ionen typisch für cis4-tert-Butyl-methoxycyclohexan und wird durch eine $H$-Wanderung von der Seitenkette zur OR-Gruppe erklärt ${ }^{9)}$. An Molekül-Modellen läßt sich nachweisen, daß bei den Molekül-Ionen von 7 ebenfalls ein kleiner Abstand zwischen den Hydroxygruppen und der tert-Butylgruppe erreicht werden kann, wenn die Molekül-Ionen in die flexible Bootkonformation oder in die Sesselkonformation mit axialer Anordnung der drei Substituenten umgewandelt werden.

Die Eliminierung von $\mathrm{C}_{4} \mathrm{H}_{8}$ wird aus den Molekül-Ionen von 5 und 6 nur in sehr geringem Ausmaß beobachtet; dieses stützt die Annahme, daß ein Zusammenhang zwischen dem Eintreten dieser Fragmentierung und der besonderen räumlichen Anordnung der Substituenten in den intakten cyclischen Molekül-Ionen von 7 besteht. Bei einer $\mathrm{C}_{4} \mathrm{H}_{8}$ Abspaltung aus offenkettigen Molekül-Ionen wären nämlich vergleichbare Intensitäten für die Ionen $m / e=116$ in den Massenspektren aller Isomeren zu erwarten. Die Bildung der für 7 spezifischen Fragment-Ionen läßt sich daher nur gut erklären, wenn die MolekülIonen von 7 aus der Grundkonformation in die energiereichere Boot- und Sesselkonformation übergehen. 


\section{b) Dimethyläther}

Übersichtlicher als bei den freien Diolen läßt sich der Einfluß der Molekülgeometrie und der Konformationen in den Massenspektren der Dimethyläther 12-14 verfolgen (Abb. 2). Die Molekül-Ionen von 12 besitzen in der Grundkonformation durch die diaxiale Stellung beider Methoxygruppen eine sterisch günstige Anordnung für eine reaktive Wechselwirkung zwischen diesen Substituenten, die durch Eliminierung von Formaldehyd und anschließende Abspaltung von Methanol zu den Ionen $m / e=170$ und 138 führt. In der Tat ist im Spektrum von 12 ein Peak bei $m / e=170$ vorhanden und der Peak 138 dominiert im oberen Massenbereich. Die Intensität der Ionen $\left[\mathrm{M}-\mathrm{CH}_{3} \mathrm{OH}\right]^{+}$mit $m / e=168$ ist dagegen relativ gering, ebenso die Intensitäten der daraus entstehenden Ionen $m / e=153\left(168-\mathrm{CH}_{3}\right), 125\left(168-\mathrm{C}_{3} \mathrm{H}_{7}\right), 121\left(168-\mathrm{C}_{2} \mathrm{H}_{7} \mathrm{O}\right)$ und $111(168$ $-\mathrm{C}_{4} \mathrm{H}_{9}$ ).
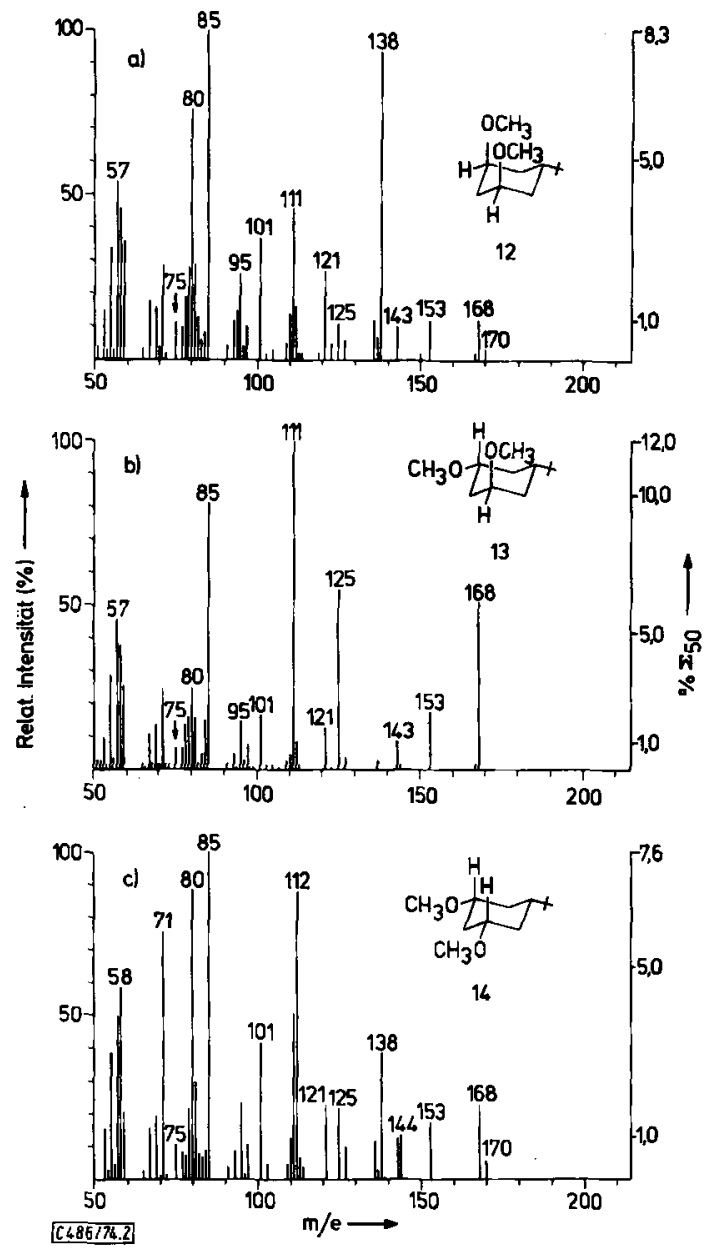

Abb. 2. Massenspektren der 5-tert-Butyl-1,3-dimethox ycyclohexane, $70 \mathrm{eV},{ }^{13} \mathrm{C}$-korrigiert

a) $5 t$-tert-Butyl-1r,3c-dimethoxycyclohexan (12)

b) $5 c$-tert-Butyl-1r,3t-dimethoxycyclohexan (13)

c) $5 c$-tert-Butyl-1r,3c-dimethoxycyclohexan (14) 
Im Massenspektrum von 12- $\mathrm{d}_{1}$ beobachtet man die Eliminierung von $\mathrm{CH}_{3} \mathrm{OH}$ und $\mathrm{CH}_{3} \mathrm{OD}$ im Verhältnis von 70:30 (Tab. 1). Eine Eliminierung von $\mathrm{CH}_{3} \mathrm{OD}$ ist aus sterischen Gründen nur nach einer Aufspaltung des Cyclohexanrings der Molekül-Ionen möglich. Da beide Carbinolgruppierungen des Molekül-Ions gleichwertig sind, entstehen somit mindestens $60 \%$ der $\left[\mathrm{M}-\mathrm{CH}_{3} \mathrm{OH}\right]^{\ddagger}$-Ionen aus offenkettigen Molekül-Ionen ohne sterische Kontrolle der Fragmentierung. Beim cis-1,3-Dimethoxycyclohexan ist für diesen sterisch nicht kontrollierten Mechanismus der Methanolabspaltung ein Anteil von $36 \%$ nachgewiesen worden ${ }^{2 a)}$.

Im Gegensatz zu 12 ist bei 13 eine reaktive Wechselwirkung zwischen den Methoxygruppen in den intakten Molekül-Ionen nicht möglich. Es entspricht daher der Erwartung, $\mathrm{da} \beta$ im Massenspektrum weder die Ionen $m / e=170$ noch 138 beobachtet werden. Das Molekül-Ion von 13 besitzt aber günstige sterische Voraussetzungen für die Abspaltung von Methanol unter Beteiligung eines H-Atoms der Carbinolgruppierung. Dies erklärt, warum die Peaks der $\left[\mathrm{M}-\mathrm{CH}_{3} \mathrm{OH}\right]^{\dagger}$-Ionen bei $m / e=168$ und ihrer Folgeprodukte bei 153, 125 und 111 das Massenspektrum beherrschen.

Die energetisch günstige 1,3-Eliminierung ist sowohl in der Grundkonformation als auch in der anderen energiereicheren Sesselkonformation von 13 mit axialer Anordnung der tert-Butylgruppe möglich. Zwischen diesen Möglichkeiten kann mit Hilfe des Massenspektrums von 13- $\mathrm{d}_{1}$ unterschieden werden, weil in der Grundkonformation das D-Atom eine axiale Position einnimmt und die Eliminierung von $\mathrm{CH}_{3} \mathrm{OD}$ erfolgt, während in der anderen Sesselkonformation das H-Atom der anderen Carbinolgruppe in axialer Stellung für die 1,3-Eliminierung von $\mathrm{CH}_{3} \mathrm{OH}$ bereitsteht. Man beobachtet im Massenspektrum von 13- $\mathrm{d}_{1}$ eine Aufteilung der Methanolabspaltung für $\left[\mathrm{M}-\mathrm{CH}_{3} \mathrm{OD}\right]^{\ddagger} /$ $\left[\mathrm{M}-\mathrm{CH}_{3} \mathrm{OH}\right]^{\dagger}$ im Verhältnis $9: 1$. Daraus kann man jedoch noch nicht ableiten, daß diese Fragmentierungsreaktion zu 90\% aus der Grundkonformation und $\mathrm{zu} 10 \%$ aus der energiereichen zweiten Sesselkonformation erfolgt, weil die Eliminierung von $\mathrm{CH}_{3} \mathrm{OH}$ aus 13- $\mathrm{d}_{1}$ mehrdeutig ist und andere Mechanismen als die 1,3-Eliminierung an ihr beteiligt sind. Außerdem kann $\left[\mathrm{M}-\mathrm{CH}_{3} \mathrm{OH}\right]^{ \pm}$andere Strukturen besitzen und mit anderer Wahrscheinlichkeit weiterzerfallen als $\left[\mathrm{M}-\mathrm{CH}_{3} \mathrm{OD}\right]^{+}$, so daß aus dem Intensitätsverhältnis dieser Ionen nicht ohne weiteres quantitative Schlüsse auf die Beteiligung der Konformeren an der Eliminierung gezogen werden können. Die Abspaltung von Methanol aus den Molekül-Ionen von 12 und 14 erfolgt wegen der ungünstigen sterischen Verhältnisse mit hoher Wahrscheinlichkeit nach einer Aufsprengung des Cyclohexanringes. Man kann hilfsweise annehmen, daß ein entsprechender Anteil der $\left[\mathrm{M}-\mathrm{CH}_{3} \mathrm{OH}\right]^{+}$-Ionen am Gesamt-Ionenstrom wie bei $12(1.0 \%)$ oder $14(1.7 \%)$ auch bei 13 auf diesem Wege entsteht. Bei einem Anteil der $\left[\mathrm{M}-\mathrm{CH}_{3} \mathrm{OH}\right]^{\ddagger}$-Ionen von 13 am Gesamt-Ionenstrom von $6.1 \%$ errechnet sich daraus, daß bei 13 zwischen 72 und $84 \%$ aller $\left[\mathrm{M}-\mathrm{CH}_{3} \mathrm{OH}\right]^{\dagger}$ Ionen durch eine stereospezifische 1,3-Eliminierung aus intakten cyclischen MolekülIonen entstehen. Geht man andererseits davon aus, daß im Massenspektrum von 13- $\mathrm{d}_{1}$ die $10 \%\left[\mathrm{M}-\mathrm{CH}_{3} \mathrm{OH}\right]^{\ddagger}$-Ionen aus offenkettigen Molekül-Ionen ohne sterische Kontrolle entstehen, so müssen wegen der Gleichwertigkeit beider Carbinolgruppierungen in den offenkettigen Molekül-Ionen zusätzlich auch $10 \%\left[\mathrm{M}-\mathrm{CH}_{3} \mathrm{OD}\right]^{\dagger}$-Ionen auf diesem Wege gebildet werden. Die verbleibenden $80 \%\left[\mathrm{M}-\mathrm{CH}_{3} \mathrm{OD}\right]^{+}-$-Ionen stimmen gut mit dem Anteil von $72-84 \%$ an den durch Methanolabspaltung gebildeten FragmentIonen überein, die aus intakten Molekül-Ionen entstehen können. Dies bedeutet aber, 
daß bei 13 eine sterisch kontrollierte Methanolabspaltung ausschließlich von MolekülIonen in der Grundkonformation ausgeht und eine Beteiligung anderer Konformationen an den Fragmentierungen nicht nachzuweisen ist.

Im Massenspektrum des Dimethyläthers 14 ist von Interesse, daß hier ebenso wie im Spektrum des Isomeren 12 deutliche Peaks für die Ionen $\left[\mathrm{M}-\mathrm{CH}_{2} \mathrm{O}\right]^{\dagger}$ bei $m / e=170$ und $\left[\mathrm{M}-\mathrm{CH}_{2} \mathrm{O}-\mathrm{CH}_{3} \mathrm{OH}\right]^{ \pm}$bei 138 gefunden werden. Eine reaktive Wechselwirkung zwischen den Methoxysubstituenten, die zur Bildung dieser Ionen führt, ist in der Grundkonformation von 14 nicht möglich, sondern erfordert ein Umklappen der MolekülIonen in die energiereichere zweite Sesselkonformation, zumindest aber eine Umwandlung in die flexible Bootkonformation. Der merkliche Intensitätsabfall dieser 138-Ionen bei 14 im Vergleich zu 12, das bereits in der Grundkonformation optimale Voraussetzungen für diese Fragmentierungen besitzt, ist daher verständlich. Konformationsänderungen der Molekül-Ionen von 14 vor èinem Zerfall müssen jedoch möglich sein. Dafür spricht auch die Bildung der Ionen $m / e=144$ und 112 durch Abspaltung von $\mathrm{C}_{4} \mathrm{H}_{8}$ bzw. $\left[\mathrm{C}_{4} \mathrm{H}_{8}+\right.$ $\left.\mathrm{CH}_{3} \mathrm{OH}\right]$ aus den Molekül-Ionen, die mit hoher Intensität nur bei 14 erfolgt. Wie bei dem freien Diol 7 können diese Fragmentierungen durch eine $\mathrm{H}$-Wanderung von der tertButylseitenkette zu einer der Methoxygruppen in der Boot- oder energiereicheren Sesselkonformation erklärt werden:

Schema 6

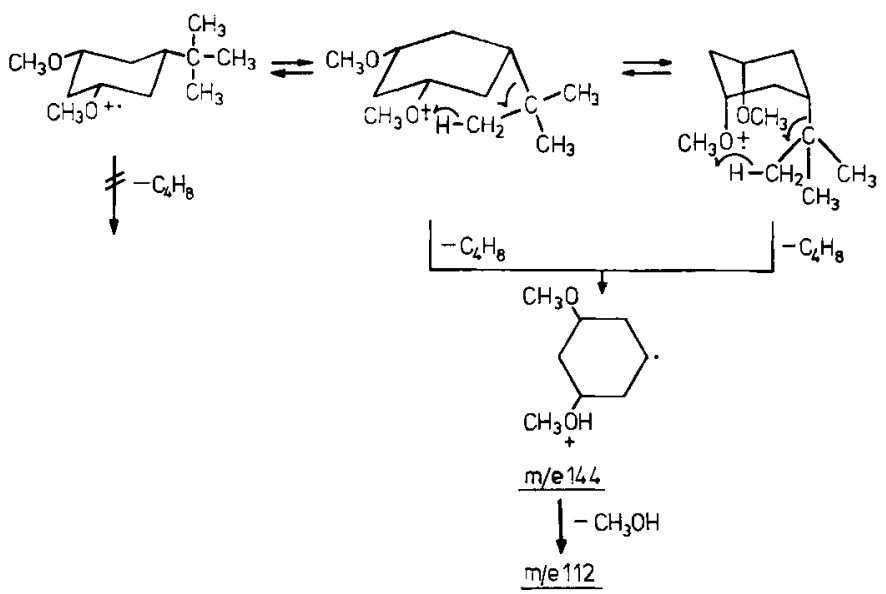

Im Gegensatz zu 13, für das Konformationsänderungen der Molekül-Ionen vor der Fragmentierung ausgeschlossen werden können, läßt sich das Fragmentierungsmuster im Massenspektrum von 14 daher nur erklären, wenn die Molekül-Ionen vor der Fragmentierung von der Grundkonformation in energiereichere Konformationen übergehen.

\section{c) Monomethyläther}

Die Massenspektren der 4 isomeren Monomethyläther des 5-tert-Butyl-1,3-cyclohexandiols unterscheiden sich ebenfalls beträchtlich (Abb. 3). Im Spektrum von 8 mit axialer Stellung der $\mathrm{CH}_{3} \mathrm{O}$ - und der HO-Gruppe treten nur kleine Peaks für die Ionen [M - 
$\left.\mathrm{H}_{2} \mathrm{O}\right]^{ \pm}$bei $m / e=168$ und $\left[\mathrm{M}-\mathrm{CH}_{3} \mathrm{OH}\right]^{\dagger}$ bei 154 auf. Die Analyse der Massenspektren deuterierter Derivate ergibt (Tab. 1), daß der größere Anteil der Ionen $\left[\mathrm{M}-\mathrm{CH}_{3} \mathrm{OH}\right]^{\ddagger}$ durch Reaktion zwischen axialer Methoxy- und Hydroxygruppe in der Grundkonformation gebildet wird. Die so entstandenen Ionen $m / e=154$ zerfallen wie beim Diol 5 durch eine McLafferty-Umlagerung zu Ionen $m / e=110$. Der erste größere Peak im Spektrum von 8 bei $m / e=138$ entsteht durch die Reaktionsfolge $\mathrm{M}^{\dagger} \rightarrow\left[\mathrm{M}-\mathrm{CH}_{2} \mathrm{O}-\mathrm{H}_{2} \mathrm{O}\right]^{\dagger}$ und geht ebenfalls auf eine Reaktion zwischen beiden axialen Substituenten in der Grundkonformation der Molekül-Ionen zurück.

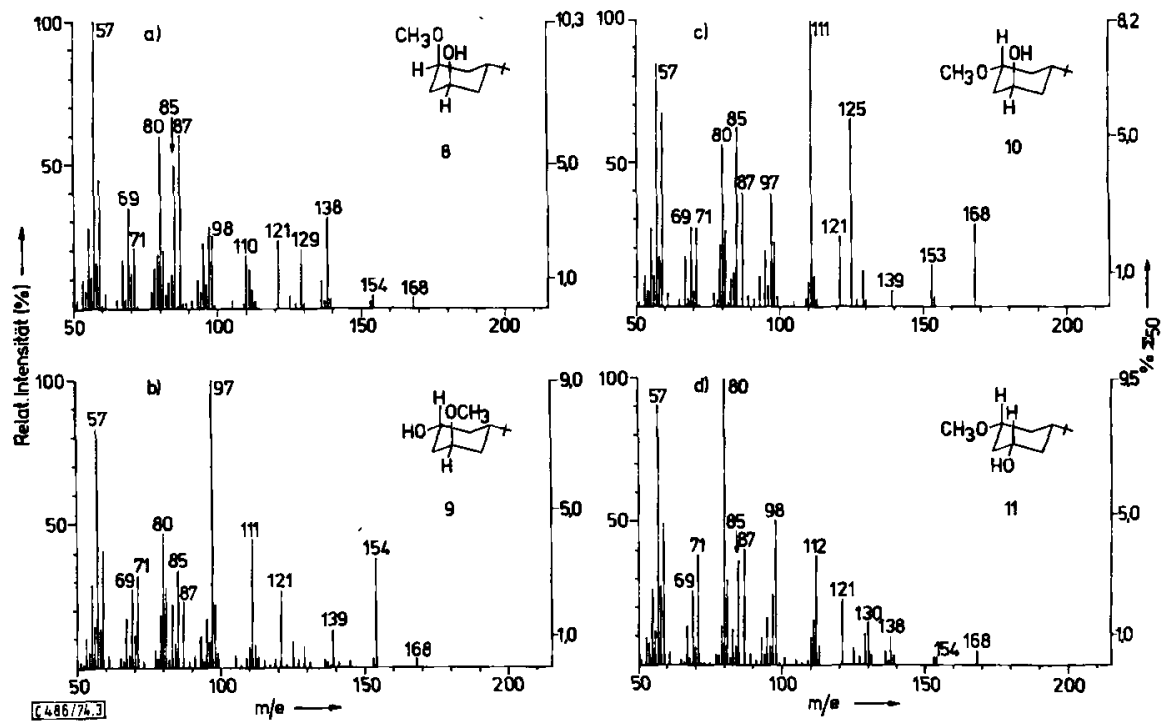

Abb. 3. Massenspektren der 5-tert-Butyl-3-methox ycyclohexanole, $70 \mathrm{eV},{ }^{13} \mathrm{C}$-korrigiert

a) $5 t$-tert-Butyl-3c-methoxy-1r-cyclohexanol (8)

b) $5 c$-tert-Butyl-3t-methoxy-1r-cyclohexanol (9)

c) $5 t$-tert-Butyl-3t-methoxy-1r-cyclohexanol (10)

d) Sc-tert-Butyl-3c-methoxy-1r-cyclohexanol (11)

Die Unterschiede in den Massenspektren der beiden Monomethyläther 9 und 10 sind besonders interessant. Bei 9 mit axialer Methoxygruppe und äquatorialer Hydroxygruppe beobachtet man bei $m / e=154$ einen großen Peak für die Ionen $\left[\mathrm{M}-\mathrm{CH}_{3} \mathrm{OH}\right]^{\dagger}$, ebenso treten die Peaks der Folgeprodukte dieser Ionen bei $m / e=139,111$ und 97 deutlich hervor. Die Intensität der Ionen $\left[\mathrm{M}-\mathrm{H}_{2} \mathrm{O}\right]^{ \pm}$bei $m / e=168$ ist dagegen sehr gering. Das Massenspektrum von $10 \mathrm{mit}$ axialer Hydroxygruppe und äquatorialer Methoxygruppe wird umgekehrt von den Ionen $\left[\mathrm{M}-\mathrm{H}_{2} \mathrm{O}\right]^{\dagger}$ und deren Folgeprodukten bei $m / e=153,125$ und 111 beherrscht, während der Peak für die Ionen $\left[\mathrm{M}-\mathrm{CH}_{3} \mathrm{OH}\right]^{\dagger}$ jetzt sehr klein ist. Aus 9 und 10 wird daher ohne Rücksicht auf die Natur der OR-Gruppe sehr stark bevorzugt derjenige Substituent abgespalten, der in der Grundkonformation des Molekül-Ions eine axiale Stellung und damit eine günstige Anordnung für die sterisch kontrollierte 1,3-Eliminierung besitzt. Dies wird auch durch das Massenspektrum von 9- $\mathrm{d}_{1}$ bestätigt, in dem die Eliminierung von $\mathrm{CH}_{3} \mathrm{OH}$ aus den Molekül-Ionen zu mehr als 
95\% durch die Abspaltung von $\mathrm{CH}_{3} \mathrm{OD}$ ersetzt ist (Tab. 1). Diese Ergebnisse zeigen, daB die Molekül-Ionen von 9 und 10 wie die des entsprechenden Dimethyläthers 13 bevorzugt aus der Grundkonformation fragmentieren und eine Umwandlung in andere Konformationen nur in einem sehr geringen Ausmaß, wahrscheinlich aber gar nicht eintritt.

Der Monomethyläther 11 liefert im Massenspektrum wie 8 bei $m / e=138$ einen Peak für $\left[\mathrm{M}-\mathrm{CH}_{2} \mathrm{O}-\mathrm{H}_{2} \mathrm{O}\right]^{+}$-Ionen, jedoch mit deutlich geringerer Intensität, und bei $m / e=130$, 112 und 98 charakteristische Peaks der Ionen $\left[\mathrm{M}-\mathrm{C}_{4} \mathrm{H}_{8}\right]^{ \pm},\left[\mathrm{M}-\mathrm{C}_{4} \mathrm{H}_{8}-\mathrm{H}_{2} \mathrm{O}\right]^{+}$und $\left[\mathrm{M}-\mathrm{C}_{4} \mathrm{H}_{8}-\mathrm{CH}_{3} \mathrm{OH}\right]^{\ddagger}$. Die Bildung dieser Fragment-Ionen mit einer für 11 typischen größeren Intensität läßt sich wie bei dem entsprechenden Dimethyläther 14 wieder nur erklären, wenn der Zerfall der Molekül-Ionen nicht nur in der Grundkonformation eintritt, sondern vorher eine Konformationsänderung zur Bootkonformation oder zur zweiten energiereicheren Sesselkonformation erfolgt ist.

\section{Diskussion}

Die Analyse der Massenspektren der Stereoisomeren der 5-tert-Butyl-1,3-cyclohexandiole, 5-tert-Butyl-3-methoxycyclohexanole und 5-tert-Butyl-1,3-dimethoxy-cyclohexane führt zu dem zunächst widersprüchlichen Ergebnis, daß bei den Derivaten mit axialäquatorialer Anordnung der OR-Gruppen keine Konformationsänderung der MolekülIonen vor der Fragmentierung eintritt, während bei einer diäquatorialen Stellung der ORSubstituenten Konformationsänderungen der Molekül-Ionen für die massenspektrometrischen Abbaureaktionen notwendig sind. Bei den Verbindungen mit diaxialen ORSubstituenten in der Grundkonformation kann eine Umwandlung der Molekül-Ionen in die Bootkonformation vor der Fragmentierung nicht ausgeschlossen werden, ist aber zur Deutung der Massenspektren nicht notwendig.

Bevor eine Erklärung für das gegensätzliche Verhalten der Molekül-Ionen der Verbindungen 6 und 7,9 bzw. 10 und 11 sowie 13 und 14 gesucht wird, ist zu prïfen, ob nicht bereits die neutralen Moleküle dieser Verbindungen unter den Meßbedingungen Konformationsänderungen erleiden. Die Enthalpiedifferenzen bei $25^{\circ} \mathrm{C}$ zwischen der als Grundkonformation angenommenen Sesselkonformation A mit äquatorialen tert-Butylsubstituenten und der Bootkonformation B bzw. der zweiten Sesselkonformation C lassen sich nach einem Verfahren von Turner ${ }^{11)}$ abschätzen und sind in Tab. 2 angegeben.

Schema 7

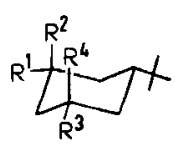

A

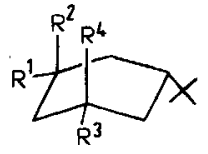

B

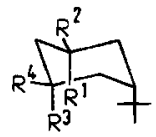

C

\begin{tabular}{|c|c|c|c|c|c|c|}
\hline & & & $\mathbf{R}^{1}$ & $R^{2}$ & $\mathbf{R}^{3}$ & $R^{4}$ \\
\hline 5 & 8 & 12 & $H$ & $\mathrm{OR}$ & $\mathrm{H}$ & OR \\
\hline 6 & 9 & 1013 & $\mathrm{OR}$ & $\mathrm{H}$ & $\mathbf{H}$ & OR \\
\hline 7 & 11 & 14 & $O R$ & $\mathrm{H}$ & OR & $H$ \\
\hline
\end{tabular}

${ }^{11)}$ R. B. Turner, J. Amer. Chem. Soc. 74, 2118 (1952). 
Tab. 2. Enthalpiedifferenzen der Konformationen von 5-tert-Butyl-1,3-cyclohexandiolen

\begin{tabular}{llllll}
\hline & Verbindung & \multicolumn{2}{c}{$\begin{array}{c}\text { Enthalpiedifferenz }(\mathrm{kcal} / \mathrm{mol}) \\
\mathbf{B}-\mathbf{A}\end{array}$} & $\mathbf{C}-\mathbf{A}$ \\
\hline $\mathbf{5}$ & $\mathbf{8}$ & $\mathbf{1 2}$ & $\geqslant 3.6$ & $\geqslant 3.6$ \\
$\mathbf{6}$ & 9 & $\mathbf{1 0}$ & $\mathbf{1 3}$ & $>5.4$ & $>6.6$ \\
7 & $\mathbf{1 1}$ & $\mathbf{1 4}$ & $>7.2$ & $>9.6$ \\
\hline
\end{tabular}

Die kleinste Enthalpiedifferenz von $3.6 \mathrm{kcal} / \mathrm{mol}$ liegt bei dem Isomeren 5 (bzw. 8 oder 12) zwischen den Konformationen $\boldsymbol{A}$ und $B$ vor. Sie reicht aber immer noch aus, um bei $25^{\circ} \mathrm{C}$ ein Populationsverhältnis von $600: 1$ für 3A : 3B einzustellen. Diese geringe Konzentration an 3B ist für das Fragmentierungsmuster des Massenspektrums ohne Bedeutung, wenn die Enthalpiedifferenz zwischen $\mathbf{A}$ und $\mathbf{B}$ auch bei den erhöhten Temperaturen im Massenspektrometer von $170-250^{\circ} \mathrm{C}$ noch besteht.

Die bewegliche Bootkonformation $\mathbf{B}$ ist gegenüber den Sesselkonformationen $\mathbf{A}$ und $\mathbf{C}$ bei zunehmender Temperatur durch den Einfluß der Entropie begünstigt. Ob dieser Effekt ausreicht, um bei den Temperaturen im Massenspektrometer eine merkliche Konzentration von Molekülen in der Konformation B zu erzeugen, läßt sich nicht sicher genug abschätzen, sondern nur über die Temperaturabhängigkeit der Massenspektren prüfen. Dabei müßten für die sterisch kontrollierten Fragmentierungen besondere Effekte auftreten: man erwartet z. B. für 12, daß im Vergleich zu der unabhängig von der Molekülkonformation ablaufenden Abspaltung von $\mathrm{C}_{4} \mathrm{H}_{9}$-Radikalen $\mathrm{zu}$ den Ionen $\mathrm{m} / \mathrm{e}=143$ die Intensität der Ionen $\left[\mathrm{M}-\mathrm{CH}_{2} \mathrm{O}-\mathrm{CH}_{3} \mathrm{OH}\right]^{\dagger}$ bei $m / e=138$ durch eine Verschiebung des Konformationsgleichgewichtes zugunsten von 12B (und 12C) bei Temperaturerhöhung abnimmt, weil die Ionen $m / e=138$ bevorzugt aus Molekül-Ionen in der Konformation 12A entstehen. Umgekehrt sollte bei 14 die Intensität der Ionen $m / e=138$ relativ zu den Ionen $m / e=143$ bei einer Temperaturerhöhung zunehmen, weil in dieser Verbindung die Ionen $m / e=138$ nicht aus 14A, sondern aus 14B bzw. C gebildet werden. Verfolgt man die Massenspektren von 12 und 14 von niederen Temperaturen (EinlaßSystem $50^{\circ} \mathrm{C}$, Ionenquelle ca. $100^{\circ} \mathrm{C}$ ) zu hohen Temperaturen $\left(270 \mathrm{bzw}\right.$. ca. $\left.270^{\circ} \mathrm{C}\right)$, so beobachtet man in beiden Fällen eine geringfügige Abnahme der relativen Intensität der Ionen $m / e=138$ (Faktor ca. 0.95). Die durch eine Verschiebung der Konformationsgleichgewichte bedingten Effekte treten jedoch nicht auf, so daß sichergestellt ist, daß auch bei den erhöhten Temperaturen in der Ionenquelle die neutralen Moleküle vorwiegend die Grundkonformation A einnehmen und nur jeweils ein unbedeutender Anteil in den Konformationen $\mathbf{B}$ und $\mathbf{C}$ vorliegt. Die in den Massenspektren der Isomeren aufgefundenen Unterschiede müssen daher durch die Eigenschaften der Molekül-Ionen erklärt werden.

Den Schlüssel zum Verständnis des unterschiedlichen Verhaltens der Moleküil-Ionen des 5c-tert-Butyl-1r,3t-cyclohexandiols (6) und seiner Derivate 9, 10 und 13 auf der einen Seite und der Molekül-Ionen des 5c-tert-Butyl-1r,3c-cyclohexandiols (7) und seiner Derivate 11 und 14 auf der anderen Seite liefert die Tatsache, daß massenspektrometrische Fragmentierungsreaktionen sehr schnell ablaufen. So muß die Bildung von Ionen, die zu den Peaks in einem „normalen“, durch Elektronenstoß erzeugten Massenspektrum führen, innerhalb von $10^{-6} \mathrm{~s}$ abgeschlossen sein. Fragment-Ionen, die in ,normalen“ Massenspektren bei der Feldionisation beobachtet werden, müssen sogar in der noch kürzeren 
Zeit von $\leqslant 10^{-10}$ s entstanden sein ${ }^{12}$. Diese sehr schnellen Reaktionen sind typisch für Fragmentierungen durch einen einfachen Bindungsbruch, es sind aber auch sehr schnelle Umlagerungsreaktionen der Molekül-Ionen bekannt ${ }^{13)}$. $\mathrm{Zu}$ diesen schnellen Eliminierungsreaktionen gehört die Wasserabspaltung aus den Molekuil-Ionen aliphatischer Alkohole.

Die Umwandlung der Sessel- und Bootkonformationen von Cyclohexanderivaten ineinander erfolgt in ca. $10^{-8} \mathrm{~s}^{14)}$, so daß Konformationsänderungen und massenspektrometrische Fragmentierungen bei diesen Verbindungen vergleichbare Geschwindigkeiten besitzen können. Die charakteristischen Fragmentierungsreaktionen der Cyclohexandiole und ihrer Methyläther, die einer sterischen Kontrolle unterliegen, sind die Eliminierungen von $\mathrm{H}_{2} \mathrm{O}$ bzw. $\mathrm{CH}_{3} \mathrm{OH}$ und $\mathrm{CH}_{2} \mathrm{O}$, deren erster Reaktionsschritt die Übertragung eines relativ locker gebundenen H-Atoms auf eine OR-Gruppe ist. Wenn diese H-Übertragung bei kleinem räumlichem Abstand der beteiligten Gruppen sehr schnell erfolgt, so wird bei den Molekül-Ionen des 5c-tert-Butyl-1r,3t-cyclohexandiols (6) und dessen Abkömmlingen 9,10 und 13, die alle in der Grundkonformation bereits günstige sterische Voraussetzungen für die 1,3-Eliminierung von $\mathrm{H}_{2} \mathrm{O}$ bzw. $\mathrm{CH}_{3} \mathrm{OH}$ besitzen, die Fragmentierung eingeleitet, bevor die beim Ionisationsproze $B$ übertragene Anregungsenergie zu einer Konformationsänderung der Molekül-Ionen führen kann. Ebenso erfolgt bei den Molekül-Ionen von $5 t$-tert-Butyl-1r,3c-cyclohexandiol (5) und seinen Derivaten 8 und 12, bei denen in der Grundkonformation ein kleiner Abstand zwischen den beiden axialen OR-Gruppen vorhanden ist, die einleitende $\mathrm{H}$-Übertragung für die Abspaltung von $\mathrm{H}_{2} \mathrm{O}$ bzw. $\mathrm{CH}_{2} \mathrm{O}$ durch Wechselwirkung zwischen beiden OR-Gruppen schneller als die Konformationsänderungen. Die Konkurrenz zwischen stereospezifischer Fragmentierung und Konformationsänderung der Molekül-Ionen von 5, 6 und den entsprechenden Derivaten geht daher zugunsten der sterisch kontrollierten Eliminierungen aus, so daß die Massenspektren dieser Verbindungen dem erwarteten Fragmentierungsmuster für Reaktionen aus der Grundkonformation entsprechen.

In den Molekül-Ionen des 5c-tert-Butyl-1r,3c-cyclohexandiols (7) und seiner Derivate 11 und 14 sind dagegen in der Grundkonformation die Voraussetzungen für schnelle sterisch kontrollierte Fragmentierungen nicht vorhanden. Das Verhalten dieser Verbindungen im Massenspektrometer zeigt, daß hier Konformationsumwandlung und Fragmentierung durch sterisch unspezifische Reaktionen miteinander konkurrieren. Wird im Laufe der Konformationsänderungen jedoch eine Konformation der Molekül-Ionen erreicht, in der eine schnelle reaktive Wechselwirkung zwischen den beiden OR-Gruppen oder einer der OR-Gruppen und dem tert-Butylsubstituenten möglich ist, so erfolgt Abspaltung von $\mathrm{H}_{2} \mathrm{O}, \mathrm{CH}_{2} \mathrm{O}$ oder $\mathrm{C}_{4} \mathrm{H}_{8}$. Die Fragment-Ionen im Massenspektrum von 7 und seinen Derivaten stammen daher aus Molekül-Ionen mit allen drei Konformationen $\mathbf{A}, \mathbf{B}$ und $\mathbf{C}$.

Die Tatsache, daß Konformationsänderungen und Fragmentierungen der MolekülIonen Prozesse mit vergleichbaren Geschwindigkeiten sind, ist bisher bei der Deutung der Massenspektren stereoisomerer Verbindungen wenig beachtet worden. Nach der Quasi-

${ }^{12)}$ H. D. Beckey, Field Ionisation Mass Spectrometry, Pergamon Press, Oxford-New York-Toronto-Sydney 1971.

13) Zit. ${ }^{12)}$, S. 163 ff.

14) F. R. Jensen, D. S. Noyce, C. H. Sederholm und A. J. Berlin, J. Amer. Chem. Soc. 84, 386 (1962). 
Gleichgewichtstheorie der Massenspektren ${ }^{15)}$ nimmt die Geschwindigkeit einer massenspektrometrischen Fragmentierung mit der überschüssigen Anregungsenergie der Molekül-Ionen schnell zu. Wenn daher für den Eintritt einer sterisch kontrollierten Fragmentierung eine Konformationsänderung der Molekül-Ionen notwendig ist, so darf der Energieunterschied zwischen der Konformation des Übergangszustandes und der Grundkonformation nicht $\mathrm{zu}$ groß sein, weil sonst eine Fragmentierung der Molekül-Ionen durch unspezifische Reaktionen bevorzugt wird. Außerdem muß die „Frequenz“, mit der die Konformation des Übergangszustandes eingenommen wird, genügend groß sein, damit innerhalb der Aufenthaltszeit der Molekül-Ionen in der Ionenquelle ein ausreichender Anteil der Ionen diese Konformation erreichen kann. Bei den bisher untersuchten Derivaten

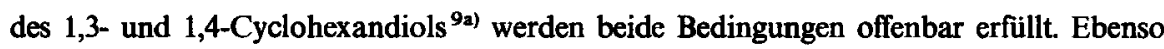
findet man in den Massenspektren der Derivate des 1,3-16) und 1,4-Decalindiols ${ }^{17)}$ die erwarteten sterisch kontrollierten Fragmentierungen, wenn eine Konformation mit kurzem Abstand zwischen den beiden Carbinolgruppen möglich ist. Während man aber im Massenspektrum des Dimethyläthers des cis-1,5-Decalindiols (15) eine Abspaltung von $\mathrm{CH}_{2} \mathrm{O}$ durch reaktive Wechselwirkungen zwischen den Methoxygruppen in den Molekül-Ionen beobachtet ${ }^{18)}$, können im Massenspektrum des Dimethyläthers von cis-2,7-Decalindiol (16) keine Anzeichen für sterisch kontrollierte $\mathrm{CH}_{2} \mathrm{O}$ - und $\mathrm{CH}_{3} \mathrm{OH}$ Abspaltungen gefunden werden ${ }^{16)}$ (Schema 8). Bei 15 ist ein kleiner Abstand zwischen den Methoxygruppen in einer "normalen“ Konformation des Decalingerüstes mit beiden Ringen in der Sesselform und axialen OR-Substituenten vorhanden, während bei 16 ein geringer Abstand zwischen den Methox ygruppen nur in einer Konformation erreicht wird, bei der einer der Ringe in einer Bootkonformation vorliegt. Offenbar ist die Wahrscheinlichkeit, daß die Molekül-Ionen von 16 innerhalb ihrer kurzen Aufenthaltszeit in der Ionenquelle diese spezielle Konformation einnehmen, zu gering für nachweisbare Reaktionen zwischen den Substituenten.

Schema 8

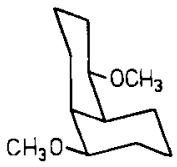

4

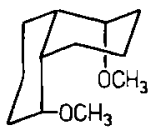

15
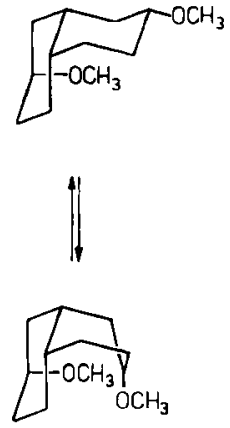

16

Dem Fonds der Chemischen Industrie danken wir für die Unterstützung dieser Arbeit.

15) H.M.Rosenstock und $M$. Kraus, Mass Spectrometry of Organic Ions (Herausgeber $F . W$. McLafferty), Academic Press, New York 1963.

16) G. Tolkien, Diplomarbeit, Univ. Hamburg 1974.

17) H. F. Grützmacher und K. H. Fechner, Org. Mass Spectrom. 7, 573 (1973).

18) H. F. Grützmacher und K. H. Fechner, Org. Mass Spectrom. 9, 152 (1974). 


\section{Experimenteller Teil}

Die Schmelzpunkte sind unkorrigiert. - Dünnschichtchromatographie: kieselgel-beschichtete Aluminiumfolien (Merck DC-Alu-Folie Kieselgel 60 F 254), Sprühreagenz Anisaldehyd-Reagenz nach Stahl ${ }^{19)}$ ( $50 \mathrm{ml}$ Essigsäure, $1.0 \mathrm{ml} \mathrm{konz.} \mathrm{Schwefelsäure,} 0.5 \mathrm{ml}$ Anisaldehyd, Nachbehandlung durch kurzzeitiges Erhitzen auf $120-130^{\circ} \mathrm{C}$ ). - IR-Spektren: Perkin-Elmer 257 Grating InfraredSpektrophotometer. - Massenspektren: CH7-Massenspektrometer Varian MAT in Verbindung mit einer Datenverarbeitungsanlage Spectrosystem TM 100 MS Varian MAT, Elektronenenergie $70 \mathrm{eV}$, Hochtemperatureinlaßsystem $150^{\circ} \mathrm{C}$. Der TemperatureinfluB auf die Massenspektren wurde mit einem CH4-Massenspektrometer, Varian MAT untersucht.

5-tert-Butyl-2-cyclohexen-1-ol; Isomerengemisch 3/4: $133 \mathrm{~g}$ 4-tert-Butylcyclohexen wurden mit $N$-Bromsuccinimid in Allylstellung bromiert ${ }^{5}$. Das Rohbromid (ca. $210 \mathrm{~g}$ ) wurde zum Gemisch $3 / 4$ hydrolysiert. Ausb. $75 \mathrm{~g}(50 \%)$.

trans-5-tert-Butyl-2-cyclohexen-1-ol (3): $40 \mathrm{~g}$ Gemisch 3/4 wurden in das Gemisch der p-Nitrobenzoesäureester übergeführt und der Ester von 3 durch fraktionierte Kristallisation isoliert. Anschließende Verseifung ergab $14 \mathrm{~g}(34 \%) 3$, Schmp. $24^{\circ} \mathrm{C}$ (Lit. ${ }^{5)} 27-28^{\circ} \mathrm{C}$ ).

cis-5-tert-Butyl-2-cyclohexen-1-ol (4): $31 \mathrm{~g}$ Gemisch 3/4 werden mit Jones-Reagenz zu $26 \mathrm{~g}$ (84\%) 5-tert-Butyl-2-cyclohexen-1-on oxidiert. Reduktion des Ketons mit $\mathrm{LiAlH}_{4}$ ergab $22 \mathrm{~g}$ ( $83 \%$, bezogen auf das Keton) 4 , Sdp. $114^{\circ} \mathrm{C} / 20$ Torr.

5t-tert-Butyl-1r,3c-cyclohexandiol (5): Reduktive Oxymercurierung ${ }^{7)}$ von $2.2 \mathrm{~g} 3$ lieferte $1.8 \mathrm{~g}$ (73\%) rohes 5, das mit Acetanhydrid/Pyridin in das Diacetat übergeführt wurde. Säulenchromatographie (Kieselgel, Benzol/Aceton $1: 1$ ) von $2.6 \mathrm{~g}$ des rohen Diacetats ergab $950 \mathrm{mg}$ des Diacetats von 5 , dessen Hydrolyse $450 \mathrm{mg}\left(18 \%\right.$, bezogen auf 3) reines 5 lieferte. Schmp. $52-53^{\circ} \mathrm{C}$ (Lit. ${ }^{7}$ $\left.53-54^{\circ} \mathrm{C}\right)$.

5c-tert-Butyl-1r,3t-cyclohexandiol (6): Analog 5 aus 2.0 g 4. Ausb. $630 \mathrm{mg}(28 \%$ ), Schmp. 124 bis $127^{\circ} \mathrm{C}$ (Lit. ${ }^{7)} 124-127^{\circ} \mathrm{C}$ ).

5c-tert-Butyl-1r,3c-cyclohexandiol (7): $200 \mathrm{mg} 6$ wurden in $20 \mathrm{ml}$ Wasser unter Zugabe von $300 \mathrm{mg}$ Raney-Nickel $50 \mathrm{~h}$ bei $80^{\circ} \mathrm{C}$ gerührt ${ }^{6}$. Die entstandene Lösung enthielt 6 und $7 \mathrm{im}$ Verhältnis von ca. 2 : 3. Nach Abtrennen des Katalysators und Eindampfen wurde das Diolgemisch durch Säulenchromatographie $(70 \mathrm{~g}$ Kieselgel nach Hermann) getrennt. Zunächst wurde mit 2 Liter Benzol/Aceton (2:1), dann mit 2 Liter Benzol/Aceton (1:1) eluiert. Neben $60 \mathrm{mg} 6$ wurden $90 \mathrm{mg}$ $7(64 \%)$ erhalten. Schmp. $143^{\circ} \mathrm{C}\left(\right.$ Lit. $\left.^{20)} 130-140^{\circ} \mathrm{C}\right)$. - IR (KBr): $3250,2920,1365,1110,1055$, $1005,855 \mathrm{~cm}^{-1}$.

5t-tert-Butyl-3c-methoxy-1r-cyclohexanol (8) und 5c-tert-Butyl-3t-methoxy-1r-cyclohexanol (9): $11.8 \mathrm{~g} 3$ wurden mit Diazomethan/BF 3 -Ätherat methyliert und der reduktiven Oxymercurierung ${ }^{7}$ unterworfen. Es wurden $11.5 \mathrm{~g}$ Isomerengemisch $8 / 9$ (ca. $4: 1$ ) erhalten. Die Trennung erfolgte durch Säulenchromatographie (140 g Kieselgel nach Hermann) in 4 Portionen. Eluierung mit Benzol/Äther ( $3: 1)$ lieferte $6.2 \mathrm{~g}(39 \%) 8, \mathrm{Sdp} .245-246^{\circ} \mathrm{C}, n_{\mathrm{D}}^{24}=1.4603$. - IR (Film): 3530,2940 , $1365,1120,1075,990,900,775 \mathrm{~cm}^{-1}$.

$$
\mathrm{C}_{11} \mathrm{H}_{22} \mathrm{O}_{2} \text { (186.3) Ber. C } 70.92 \mathrm{H} 11.90 \text { Gef. C } 71.87 \text { H } 11.93
$$

Anschließendes Eluieren mit Benzol/Äther $(1: 1)$ ergab $1.4 \mathrm{~g}(8.9 \%) 9, n_{D}^{22}=1.4690$. - IR (Film): 3350, 2940, 1365, 1235, 1110, 1085, 1055, 1010, $800 \mathrm{~cm}^{-1}$.

$$
\mathrm{C}_{11} \mathrm{H}_{22} \mathrm{O}_{2} \text { (186.3) Ber. C } 70.92 \mathrm{H} 11.90 \text { Gef. C } 70.87 \mathrm{H} 11.97
$$

19) E. Stahl, Dünnschichtchromatographie, 2. Aufl., Springer Verlag, Berlin-Heidelberg-New York 1967.

20) E. Dunkelblum, R. Levene und J. Klein, Tetrahedron 28, 1009 (1972). 
5t-tert-Butyl-3t-methoxy-1r-cyclohexanol (10): Analog 8 wurden aus $20 \mathrm{~g} 418.1 \mathrm{~g}$ Isomerengemisch erhalten. Die Reinigung erfolgte durch Säulenchromatographie in 4 Portionen $(350 \mathrm{~g}$ Kieselgel nach Hermann). Zunächst wurde mit Benzol/Äther (2:1) eluiert und das Eluat verworfen. Anschließendes Eluieren mit Benzol/Äther (1:1) lieferte $8.5 \mathrm{~g} \mathrm{(35 \% )} \mathrm{10,} n_{\mathbb{D}}^{22}=1.4697$. - IR (Film): 3400, 2940, 1365, 1140, 1095-1080, 1005, 940, 820, 770, $675 \mathrm{~cm}^{-1}$.

$$
\mathrm{C}_{11} \mathrm{H}_{22} \mathrm{O}_{2} \text { (186.3) Ber. C } 70.92 \mathrm{H} 11.90 \text { Gef. C } 71.37 \mathrm{H} 12.01
$$

$5 c$-tert-Butyl-3c-methoxy-1r-cyclohexanol (11): $5.0 \mathrm{~g} 10$ wurden mit Jones-Reagenz zum 5-tertButyl-3-methoxycyclohexanon (4.5 g Rohketon) oxidiert und durch Säulenchromatographic (350 g Kieselgel nach Hermann, Eluierung mit Benzol/Äther 6:1 und $3: 1) 3.7 \mathrm{~g}$ reines Keton erhalten. Reduktion mit $\mathrm{LiAlH}_{4}$ ergab $2.7 \mathrm{~g}$ eines Isomerengemisches aus 10 und 11 (ca. $1: 9$ ), die durch Säulenchromatographie (350 g Kieselgel nach Hermann, Eluierung mit Äther/Aceton 20:1) $1.0 \mathrm{~g}(21 \%$, bezogen auf 10) 11 neben $0.3 \mathrm{~g} 10$ lieferten. 11 : IR (Film): 3370, 2950, 1365, 1125, 1095, $1055,1011,985,960,900,850 \mathrm{~cm}^{-1}$.

$$
\mathrm{C}_{11} \mathrm{H}_{22} \mathrm{O}_{2} \text { (186.3) Ber. C } 70.92 \mathrm{H} 11.90 \text { Gef. C } 71.95 \mathrm{H} 12.03
$$

5t-tert-Butyl-1r,3c-dimethoxycyclohexan (12): $2.00 \mathrm{~g} 8$ wurden mit Diazomethan/BF ${ }_{3}$ - ̈̈therat methyliert. Nach Säulenchromatographie ( $140 \mathrm{~g}$ Kieselgel nach Hermann, Eluierung mit Benzol/ Äther $12:$ 1) Ausb. $0.98 \mathrm{~g}(45 \%)$, Sdp. $236^{\circ} \mathrm{C}, n_{\mathrm{D}}^{23}=1.4570$. - IR (Film): $2950,1365,1185,1120$, $1105,985,895,755 \mathrm{~cm}^{-1}$.

$$
\mathrm{C}_{12} \mathrm{H}_{24} \mathrm{O}_{2} \text { (200.3) Ber. C } 71.95 \mathrm{H} 12.08 \text { Gef. C } 71.73 \text { H } 12.02
$$

$5 c$-tert-Butyl-1r,3t-dimethoxycyclohexan (13): Analog 12 aus $2.00 \mathrm{~g} \mathrm{10}$; Eluierung mit Benzol/ Äther (4: 1). Ausb. $0.72 \mathrm{~g} \mathrm{(33 \% ),} \mathrm{Sdp.} 242^{\circ} \mathrm{C}, n_{\mathrm{D}}^{25}=1.4530$. - IR (Film): 2950, 1365, 1130, 1095, $1000,805,770 \mathrm{~cm}^{-1}$.

$$
\mathrm{C}_{12} \mathrm{H}_{24} \mathrm{O}_{2}(200.3) \text { Ber. C } 71.95 \mathrm{H} 12.08 \text { Gef. C } 72.00 \mathrm{H} 11.92
$$

$5 c$-tert-Butyl-1r,3c-dimethoxycyclohexan (14): Analog 13 aus $2.00 \mathrm{~g} \mathrm{11}$, Ausb. $0.85 \mathrm{~g}$ (40\%), Sdp. $246^{\circ} \mathrm{C}, n_{\mathrm{D}}^{24}=1.4538$. - IR (Film): $2950,1370,1130,1095,1030,1010,950,850 \mathrm{~cm}^{-1}$.

$$
\mathrm{C}_{12} \mathrm{H}_{24} \mathrm{O}_{2} \text { (200.3) Ber. C } 71.95 \mathrm{H} 12.08 \text { Gef. C } 71.92 \mathrm{H} 12.04
$$

